\title{
Questions SePtember 2013
}

CPD questionnaires must be completed online via www.cpdjournals.co.za

After submission you can check the answers and print your certificate.

True (A) or false (B):

Traumatic brain injury (TBI)

1. TBI is pathophysiologically differentiated into primary and secondary injury.

2. Primary injury occurs at the time of impact and results from energy transfer to the brain causes irreversible neuronal and mechanical disruption (largely in the form of axonal stretching and tearing).

3. Within the skull cavity raised intracranial pressure may arise from cerebral oedema or an expanding space occupying lesion (e.g. subdural haematoma).

Accuracy of after-hour 'red dot' trauma radiograph triage by radiographers in a South African regional hospital

4. Regarding appendicular fracture identification, the overall accuracy of reporting by radiographers was $>90 \%$.

Paramedic rapid sequence induction (RSI) in a South African emergency medical service: A retrospective observational study

5. Hypoxaemia and hypotension in the context of traumatic head injury increase mortality by $75 \%$.

Endotracheal tube cuff pressures - the worrying reality: A comparative audit of intra-operative versus emergency intubations

6. Autopsy studies have shown that maximal damage to the trachea occurs at the site of the cuff during endotracheal intubation.

7. Tracheal stenosis typically presents immediately after extubation as an airway emergency.

The Kimberley Hospital Rule (KHR) for urgent computed tomography of the brain (CTB) in a resource-limited environment

8. The KHR is an accurate clinical guideline that serves to optimise the utilisation of CTB in a resource-limited environment while reducing the requirement for immediate CTBs by a third.

Human fascioliasis in South Africa

9. Transmission of fasciola to humans is attributable to ingestion of metacercariae present on watercress and other aquatic plants.
10. Human fascioliasis is a snail-borne disease, from snails infected via fluke eggs. shed by infected animals in their faeces.

11. Rarely, fascioliasis may present with mitral incompetence and heart failure associated with eosinophilia.

12. A raised total eosinophil count has a long differential diagnosis including allergic disorders, drug hypersensitivity and parasitic infection (and rarer causes such as neoplasia, connective tissue diseases and primary eosinophilic disorders).

The dilemma of treating major burns in South Africa

13. A major burn is defined as one exceeding $30 \%$ of the total body surface area.

14. For wound closure in extensive burns, cadaveric skin may be used as a temporary biological dressing.

15. Allografts are freely available from mortuaries, South African regulations permitting duty police officers to obtain consent from the deceased's family for allograft procurement.

16. SA is fortunate in having a well-established skin bank of such allografts.

Patterns of seatbelt use in different socioeconomic communities in the Cape Town Metropole, South Africa and The promotion of road safety by healthcare professionals in South Africa

17. Traffic injuries are the top cause of premature death in children and adolescents.

18. Babies and children under three years may be safely held in the arms of adult passengers.

Haemodialysis for post-traumatic acute renal failure (ARF) factors predicting outcome

19. ARF in trauma patients is associated with a high survival rate owing to improvements in initial resuscitation and subsequent intensive care unit care.

20. In children with renal failure following severe burn injury, early burn excision decreases the inflammatory response, thereby improving renal blood flow and in turn, reducing the severity of ARF.

\section{INSTRUCTIONS}

1. Read the journal. All the answers will be found there.

2. Go to www.cpdjournals.co.za to answer the questions.

Accreditation number: MDB001/006/01/2013 (Clinical) 